\title{
Outsourcing Clinical Trials Outside of the US
}

\section{Mo Dezfuli}

Graduate in MS Regulatory Affairs program, Krieger School of Arts \& Sciences, Advanced Academic Programs, Johns Hopkins University, USA

*Corresponding author: Mo Dezfuli, Graduate in MS in Regulatory Affairs program, Krieger School of Arts \& Sciences, Advanced Academic Programs, Johns Hopkins University, USA, Tel: 415-506-6700; E-mail: mbakhshidez@yahoo.com

Received date: Sep 07, 2017; Accepted date: Sep 21, 2017; Publish date: Oct 04, 2017

Copyright: $\odot 2017$ Dezfuli M. This is an open-access article distributed under the terms of the Creative Commons Attribution License, which permits unrestricted use, distribution, and reproduction in any medium, provided the original author and source are credited.

\begin{abstract}
Outsourcing clinical trials has been a subject of both controversy and relevance in the pharmaceutical community. It is an important part of drug development that not only reveals the effectiveness of new medications, but it also brings on such a powerful effect on public health. A definition and a brief historical overview are discussed in-depth in this article, as well as its prevalence, business strategies, and need for transparency.
\end{abstract}

Keywords: Clinical trials; Good clinical practices; Health care

\section{Introduction}

Several decades ago, using outsourcing services in clinical trials was one of the most important and debatable issues in the drug development process. In 1938, the Food, Drug, and Cosmetic (FD\&C) Act created the requirement that new, investigational drugs must be tested on human subjects for safety before being available on the United States market [1]. Foreign clinical trials were not formally addressed until 1962 in which the Food and Drug Administration (FDA) informed pharmaceutical drug sponsors that foreign clinical data-which deemed premarket drugs to be safe- could be gathered as along as long as domestic clinical data was already recorded [1].

The following year after this notification was given to pharmaceutical sponsors, the FDA began to provide oversight over clinical studies; however, the current practice of offshoring clinical trials was not regularly done until the 1990s [1], showing a gradual decrease in domestic clinical trials in the United States. The practice of outsourcing clinical trials to other countries has proven to being a more cost-effective endeavor. Under the FDA guideline of 21 CFR 312.120 , for example, pharmaceutical companies can conduct a foreign clinical study for an Investigational New Drug (IND) if the study is well-designed, in alignment with Good Clinical Practices (GCPs), and under the review and approval of an Independent Ethics Committee (IEC) [2]. As of September 2017, according to clinicaltrials.gov, about 119,648 of clinical trials are conducted outside of the US, while only 91,146 clinical trials are recorded as being performed domestically [3].

In the early 2010s, developing a new drug was very costly for pharmaceutical companies, being an estimated $\$ 1.3$ billion [4], hence the need for more less expensive clinical trials. In 2014, it was reported that drug development with research and development (R\&D) and marketing approval combined reached up to approximately $\$ 2.6$ billion [5]. Outsourcing, in definition, is the practice of contracting with another entity to perform services that are otherwise conducted inhouse. It means that companies do the practice of hiring professional contractors / vendors to conduct the research instead of their own company. Usually contract research companies (CROs) are independent organizations meant to fulfil special parts of research, which is necessary for new drug discoveries.
Outsourcing is now more common in clinical trials, but it has some advantages and disadvantages, several of which will be discussed further in this review. During 2003-2005, there was a surge in conducting clinical trials in comparison to the years 1998-2002 at 52\%; in 2012 , it was projected that the rate of outsourcing as $72 \%$ [6]. As drug companies seek new ways of working faster and attaining higher standards, clinical outsourcing is constantly changing. This gives sponsors ways to have different methods of outsourcing options; they base these methods upon the International Council for Harmonisation (ICH) E6. Sponsors are responsible for the quality and integrity of the data even if they utilize outsourcing. This issue forces sponsors to find a strategy that reduces their liabilities while using outsourcing services.

The high cost of clinical trials forces sponsors and investigators to budget more, and they seek ways to have a return on their investments. Pharmaceutical companies apply a variety of strategies to improve the efficacy of drug development, aiming to reduce their costs. In an attempt to improve and reduce both mass and fixed costs, many pharmaceutical companies have downsized internal staff and turned to outsourcing various practices in research. Typically, finding participants to engage in research and hiring researchers is very costly in a domestic sense, while utilizing them abroad is less expensive. With the advent of this process, many questions have been raised, such as its ethics, the basis of its scientific validity, and its trustworthiness. As with every practice-especially those that include participation of other individuals- its pros and cons are heavily weighed.

\section{Moving Towards Outsourcing Strategies}

A cost-effective, predictable, and integrated approach to goals is an important characteristic of this module. New research says that outsourcing reduces costs by $25 \%$ and reduces Institutional Review Board (IRB) approval cycle times; decreases rejection rates for regulatory packet approval from $42 \%$; and reduces data management cost savings of $15 \%$ per page with a concurrent $20 \%$ improvement in productivity [6]. This amount is enough to move the process off the critical path for study execution. Importantly, it is the ongoing trend towards drug development in specialized therapeutic areas, such as the field of oncology. Outsourcing can make it possible for small and medium size companies to conduct clinical trials in these areas where the company itself may not have the therapeutic area expertise and/or resources to independently develop the product(s) [6]. 


\section{Advantages, Disadvantages, and Challenges of Outsourcing}

Today, there are many pharmaceutical companies that have outsourced their R\&D projects outside of the United States, and many others are considering it. There are some clear advantages disadvantages, and challenges that need to be carefully considered.

\section{Advantages}

There are huge financial gains associated with outsourcing because costs in foreign countries are significantly lower in those areas than in the United States. In addition, the materials and actual cost of location for foreign research centers can be less expensive when they are offshored. The rules for clinical research are strict in the United States, so companies can achieve greater innovation by being allowed to engage in a broad array of testing outside of the country. Another advantage of outsourcing R\&D is that companies can build strategic research alliances with foreign companies and/or universities. Mutual interests bring together both domestic and foreign knowledge to achieve innovation [7]

\section{Disadvantages}

In any relationship, there will be challenges. It is extremely vital to have solid trust and communication between parties involved in the outsourcing process. In some foreign countries, there may be concerns in regard to the researchers' competence, as well as level of quality and timeliness of delivery. Deadlines must be firm and adhered to, and communication must be as clear and thorough as possible. Some of the issues pertaining to outsourcing are given an overview in this section.

\section{Employee morale}

There is a questionability concerning employee morale as a whole. While outsourcing has been praised as a strategy for clinical trials, there have been dwindling jobs when it comes to more skillful employees. For instance, according to the Bureau of Labor Statistics (BLS), in 2010, there was a five-percent drop in U.S. pharmaceutical manufacturing employees from 2007 [8], with international outsourcing being seen as a major factor. This reveals that those who have education, knowledge, and skills may find themselves in a smaller job market as a result of outsourcing. Thus, new solutions must be found in the business model, and expertise must still be valued over the cutting of costs.

\section{Ethical issues and past incidents}

Outsourcing clinical trials can present some issues, particularly ethical issues concerning the well-being of its participants. What is pertinent about this practice alone is that participants need to be clearly informed about the risks of the clinical trials they are in, and withholding important information from them can bring on tragic results. For instance, in March 2006, a failure occurred in a clinical trial being conducted in London. In a Phase I clinical trial, eight adult volunteers were administered a drug via intravenous infusion [9], with six of them facing incredibly adverse side effects within an hour of the administration of the drug. It is implied that the participants were unaware of such side effects, with some even throwing off their shirts due to the pain of severe headaches, backaches, and even fever [9], despite giving informed consent prior to the testing. All six were admitted to the hospital shortly thereafter, even being put in the
Intensive Care Unit (ICU) ; fortunately, they survived, but not without physical damage and trauma. If clinical trials are to be the wave of the future and are to continue, then there must be full informed consent and top priority placed on patients' lives. As of 2008, the FDA has placed priority on the well-being of human participants under $21 \mathrm{CFR}$ 312.120 [10].

\section{Other challenges}

Besides the aforementioned disadvantages and ethical issues, more challenges found in outsourcing include failing to deliver requirements on time, risking non-compliance of regulatory requirements by Contract Manufacturing Organizations (CMOs), reducing in-house manufacturing expertise in terms of resources and process knowledge, and a risk of pilferage and quality compliance. According to Bhatt [11], "FDA inspections of clinical trials have been labeled as Voluntary Action Indicated (VAI) 59\%, No Action Indicated (NAI) 40\%, and Official Action Indicated (OAI) 1\%. In For Cause inspections, the proportion of OAI is $23 \%$. In India, out of 23 site inspections, 12 (52\%) were NAI and $11(48 \%)$ were VAI".

The statistics given appear to apply to all clinical trial inspections at first, with the outsourced trials in India being an example of the high numbers of VAI statuses. While an NAI status is positive, VAI statuses show that there are violations of regulations, and $48 \%$ is a high number alone. This reveals that there is more need for quality and oversight.

\section{Legal Roles and Responsibilities of Both Parties}

The FDA defines each participant's responsibilities within cooperative manufacturing agreements based on the Code of federal Regulation (21 CFR 600.3(t) and 21 CFR 610.13). Manufacture is defined as "the responsible entity to legally manufacturing a drug" [12]. Therefore, license holders are responsible for the whole process, but based on the contract, sponsors delegate some of responsibilities. The license holder should be ensured about the CRO's compliance and report any changes in the product(s). License holders are also responsible, certifying that CROs comply with federal regulations. Because of this, sponsors look for the best outsourcing provider [12].

\section{FDA Outsourcing Regulations}

When a study is conducted under an IND but is located outside of the United States, the study still must comport with all relevant FDA regulations as if it were being conducted within the United States. A sponsor is not required to conduct a foreign clinical trial (FCT) under an IND in order to use it as support for a New Drug Application (NDA) or IND. The FDA does accept FCT data from studies not conducted under an IND if two conditions are met, which include the study being conducted with GCPs and if the FDA is able to validate such data from an onsite inspection. According to the FDA, under 21 CFR 312.120, GCPs includes standards for "providing assurance that the data and reported results are credible and accurate and that the rights, safety, and well-being of trial subjects are protected" [10].

There is also a federal regulation concerning the definition of an outsourcing facility, which is covered under the Drug Quality and Security Act, being signed into law in 2013. Besides following Current Good Manufacturing Practices (GCMPs), under section 503B of the Drug Quality and Security Act, an outsourcing facility must meet the following criteria:

1. Is engaged in the compounding of sterile drugs; 
Page 3 of 4

2. Has elected to register as an outsourcing facility;

3. Complies with all of the requirements of section 503B [13].

It is noticeable that such regulations and guidelines were implemented into law in very recent years, showing that the FDA recognizes the need for more oversight and more safety in foreign clinical trial practices besides domestic standards.

\section{Strategies}

Small companies and biotech companies have relied on outsourcing for years, while Big Pharma tended to work internally; up until recently, this was especially true for global trials. As a reminder, clinical trials are very important because it facilitates getting market approval. Historically, as drug development increased, the pressure for tighter timelines went up, and pharmaceutical companies started to have difficulties with workload, so the demand for outsourcing was ultimately increased [14]. CRO companies have become bigger than Big Pharma companies with publically-traded stocks. Gradually, experts have moved from Big Pharm companies to CROs, so the R\&D sections of pharmaceutical companies have become condensed. More and more, CROs have started to offer more services with efficient costs, so they have won the competition with pharmaceutical R\&D departments. Also, CROs try to be partners in the drug development process; this strategy has a lot of stake in both sides. For example, valuable knowledge and experiences goes to CROs, which help them to get new projects, and sponsors get trustful services, which help them to focus on other innovative activities. CROs have a significant weight of responsibility, the questionability of legal liability being upon them; there is also a possibility that sponsors may transfer some of their responsibilities to CROs, thus subjecting CROs to FDA actions [1].

Typically, pharmaceutical companies tend to get only one or two CROs as partners. In the past, after increasing problems with CROs and sponsors, sponsors thought about applying this strategy in other parts of drug development instead of just clinical trials. Outsourcing services at the early stage is far more challenging than outsourcing a clinical trial; this is because every phase of drug development is different compared to other research, and it needs a unique protocol. Clinical trials, on the other hand, usually follow a constant protocol, and it is not varied.

Outsourcing in preclinical and discovery is essential; this is because of the need to access hundreds of different research assays and services in a wide range of scientific disciplines. None of the CROs can provide all requirements. Value at the early stage is derived from finding the best individual supplier from the multitude available in the global market.

\section{Present Outsourcing Strategies}

The relationship between CRO and pharmaceutical companies is one of two types, either "preferred provider" or "strategic alliance". In the preferred provider model, the contractor will get an exclusive price, in exchange for guaranteeing a big chunk of the work to be outsourced to the provider [14]. The sponsor would then work with the CROs to determine how much of the procedures are to be followed; it is noted that sponsors are responsible for all parts of drug development. In the strategic alliance, the whole range of tasks will be done by the CRO; in contrast, both parties will be involved in a more linear partnership. They share their common business together, which goes beyond the contract commitments. This creates more a sense of collaboration in CROs in their core role.

\section{Partnerships}

Both CROs and sponsors encounter some challenges. For instance, CROs dissatisfactions include insufficient communication, poorly aligned process, micromanagement, and not involving CROs early in the drug development process On the side of the sponsors, missed timelines, quality issues, and costs are main concerns. Both sides suffer from staffing changes.

At a glance, challenges in the CRO strategy are divided in (1) cost changes (46\%), (2) staffing changes (28\%), (3) protocol changes $(8 \%)$, (4) technological changes (8\%), and (5) other (10\%) [7]. To alleviate these problems and make consistent relationships during studies, one solution is clearly defining the role and responsibilities of the sponsor and CRO. This definition may be changed based on the condition, but both sides should know their responsibilities in terms of specifics. Another point is that sponsors should want a more efficient research, not just a merely cost-effective research.

As a whole, about $49.2 \%$ companies choose strategic partnerships [7]. This system has less negative effect on small businesses, but is still considerably costly. To solve this problem, companies have used academic hospital networks as a partnership. In this way, the expenses will be reduced. Factories that contribute to overall improved strategies have defined responsibilities between CROs and sponsors, are choosing and being faithful with one outsourcing model, are training employees thoroughly, and are putting in time and investment to make the relationship work [7]. This is vital, because pharmaceutical companies of different sizes are interested in working with CROs, with approximately $77 \%$ of pharmaceutical and biotech industry companies utilizing outsourced services and operations [15].

\section{Implications and Suggestions}

\section{Future challenges of the model}

First of all, experts that work for CROs are finding themselves being slowly dropped from the discipline of drug development. In the past, experts who lost their jobs in pharmaceuticals due to downsizing ended up working for CROs, but currently, CROs are staffing with individuals who lack pharma, biotech, or and/or medical device experience. Should this trend continue, CROs will find themselves with staff that is incompetent for overall research and drug development processes. Secondly, the incentive of an outsource model should be changed from lower-cost settings to an efficient practice; otherwise, the quality will be sacrificed. Also, more experts hail from the US and Europe while many patients and clinical trial subjects live abroad, so participating foreign experts are needed for more oversight.

\section{How to strengthen strategies and relationships}

In terms of strengthening partnership strategies, partnership strategies bring more responsibilities for CROs rather than sponsors, but it is necessary to set expectation on both sides of responsibilities. Experienced management is necessary for both sponsors and CROs. Well-known, intricate communication systems should exist between both parties. Frequent (such as weekly) meetings should exist. Centralized control and communication is essential to ensure that different teams are aware of the strategies, with results and lessons 
Page 4 of 4

learned across the spectrum of activities [7]. Social media is seen as the way to educate others and communicate on a more transparent level; therefore, more efforts and investment should be put into social media that is full of reliability and reputation as online sources.

Another factor that would contribute to more effectiveness of conducting foreign trials is transparency. CROs, sponsors, and pharmaceutical companies must be transparent in communications, especially when it comes to the handling of data. If companies do not hide test results to patients' investigators, advocates and doctors, then more trust and reliability can be placed onto the overall health system. As a result, there could be more participants in FCTs due to transparency and encouragement in the practice, being unafraid of any stigmas.

\section{Conclusion}

In more recent years, the FDA has set more guidelines towards the conducting of FCTs with the formal ensuring of doing ethical practices and protecting human subjects. Despite these guidelines, the keeping up of safety and efficacy is only a part of the bigger picture, with costs and business strategies making up a significant part of the overall equation. Ethics, employee morale, advantages, and disadvantages must always be taken into account for more efficiency and improved testing. The benefits of less cost and less testing restrictions have been praised when it comes to FCTs, but accountability, liability, as well as unfortunate fatalities have become the center of controversy. Transparency is not only yearned for in terms of the safety and protection of participants in clinical trials, but is so the business end of it, with communications, responsibilities, and data being more seamless in-between pharmaceutical companies, sponsors, and CROs. CROs are independent and help oversee foreign clinical trials, but legal implications with FDA regulations and the transfer of accountability from sponsors must be always kept in consideration. CROs and sponsors play pivotal roles in outsourcing clinical trials, and they appear to be more complementary in a partnership type of strategy. One side cannot thrive without the other and they have a symbiotic relationship that can bring upon more efficacy and transparency in drug development.

In the future, CROs will be operating in different models compared to now, possibly becoming stronger strategic partners with sponsors.
The future of CROs- despite any changes or advancements in the stages of drug development- is optimal in the pharmaceutical industry. With awareness about the ethical issues and business issues that are involved in FCTs, the general public can benefit greatly from them instead of equating them with past mistakes or questionable efficacy. What the end result should be is a population being introduced to new, advanced drugs that can greatly improve healthcare.

\section{References}

1. Hull D (2017) Reining in the Commercialized Foreign Clinical Trial. J Leg Med 36: 367-401.

2. CFR-Code of Federal Regulations Title 21 (2017) U.S Food and Drug Administration.

3. Trends, Charts, and Maps (2017) Clinical Trials.gov.

4. Drug Developers Are Aggressively Changing the Way They Do R\&D (2011) Tufts Center for the Study of Drug Development.

5. Cost to Develop and Win Marketing Approval for a New Drug Is $\$ 2.6$ Billion (2014) Tufts Center for the Study of Drug Development.

6. Jones J, Minor M (2010) New, Strategic Outsourcing Models to Meet Changing Clinical Development Needs. Perspect Clin Res 1: 76-79.

7. Henderson L (2017) Outsourcing in 2017: Playing to Win. Appl Clin Trials 26.

8. Soni U, Singh M (2013) Clinical Trials Outsourcing: Good or Bad? Drug Des 2: 104.

9. Suntharalingam G, Perry MR, Ward S, Brett SJ, Castello-Cortes A, et al. (2006) Cytokine Storm in a Phase 1 Trial of the Anti-CD28 Monoclonal Antibody TGN1412. N Engl J Med 355: 1018-1028.

10. Guidance for Industry and FDA Staff (2012) U.S Food and Drug Administration.

11. Bhatt A (2011) Quality of clinical trials: A moving target. Perspect Clin Res 2: 124-128.

12. Swider MG (2011) Regulatory Challenges in Global Outsourcing FDA Perspective. Pharmaceutical Outsourcing.

13. Outsourcing Facilities (2015) U.S Food and Drug Administration.

14. Wang L, Motti EF (2015) The Increasing Shift of Clinical Trials to CROs. Pharmaceutical Outsourcing.

15. Walker N (2016) Nice Insight Outsourcing Trends in 2016. Pharmaceutical Outsourcing. 